\title{
Characteristic features of disseminated carcinomatosis of the bone marrow due to gastric cancer: The pathogenesis of bone destruction
}

\author{
HIROKI KUSUMOTO $^{1}$, MASARU HARAGUCHI ${ }^{1}$, YOKO NOZUKA ${ }^{2}$, \\ YOSHINAO ODA $^{2}$, MASAZUMI TSUNEYOSHI ${ }^{2}$ and HARUO IGUCHI ${ }^{3,4}$ \\ ${ }^{1}$ Division of Gastroenterological Surgery, National Kyushu Cancer Center; ${ }^{2}$ Department of \\ Anatomical Pathology, Faculty of Medicine, Kyushu University; ${ }^{3}$ Division of Tumor Dynamics, \\ National Kyushu Cancer Center Research Institute, Fukuoka, Japan
}

Received March 31, 2006; Accepted June 19, 2006

\begin{abstract}
Disseminated carcinimatosis of the bone marrow is accompanied by solid tumors, and gastric cancer accounts for the majority. The prognosis of this condition is poor, however, the pathogenesis for wide-spread bone lesions has yet to be elucidated. In 9 patients with gastric cancer demonstrating disseminated carcinomatosis of the bone marrow, the characteristic clinicopathological features were examined. Immunohistochemistry for receptor activator of NF- $\kappa B$ ligand (RANKL) and parathyroid hormone-related protein was also performed on gastric cancer tissue and bone marrow specimens to identify the factors responsible for the occurrence of bone lesions in patients presenting with this condition. The characteristic features of disseminated carcinomatosis of the bone marrow due to gastric cancer include a yonger patient age, an elevation of serum alkaline phosphatase and/or lactate dehydrogenase levels, wide-spread bone metastases with osteolytic bone destruction, a low incidence of hypercalcemia and a histological gastric cancer type of either signet ring cell carcinoma or poorly diffentiated adenocarcinoma. The expression of RANKL, which is one of the master regulators of osteoclastic bone resorption in bone metastasis, was also found in gastric cancer cells obtained from such patients. The RANKL expressed in gastric cancer may therefore play a critical role in the promotion of osteoclast formation, which has been suggested to be involved in the pathogenesis of bone lesions.
\end{abstract}

Correspondence to: Dr Haruo Iguchi, ${ }^{4}$ Present address: Shikoku Cancer Center, Minamiumemotomachi Ko 160, Matsuyama, Japan

E-mail: higuchi@shikoku-cc.go.jp

Key words: gastric cancer, bone marrow invasion, bone destruction, receptor activator of NF- $\mathrm{KB}$ ligand

\section{Introduction}

Bone metastases diffusely invading the bone marrow with hematological disorders [i.e., disseminated intravascular coagulation (DIC), microangiopathic hemolytic anemia, etc.] tend to be accompanied by solid tumors (1), and this condition is called disseminated carcinomatosis of the bone marrow. This condition is often caused by gastric cancer among solid tumors, although its overall incidence is rare $(2,3)$. The prognosis for this condition is extremely poor, however, the pathogenesis of this condition, namely the cause for the development of such diffuse bone metastases, has yet to be elucidated. In the present study, we examined the characteristic clinicopathological features of disseminated carcinomatosis of the bone marrow accompanied by gastric cancer based on the clinical findings of 9 cases who presented with this condition at our cancer center from 1991 to 2002. We also examined the expression of receptor activator of NF- $\mathrm{\kappa B}$ ligand (RANKL) and parathyroid hormone-related protein (PTHrP), which are known to be master regulators of osteoclastic bone resorption in bone metastasis $(4,5)$, in gastric cancer tissue and bone marrow specimens obtained from these 9 patients to identify any factors related to the pathogenesis of such diffuse bone metastases observed in this condition.

\section{Patients and methods}

Patients. Nine patients with disseminated carcinomatosis of the bone marrow associated with gastric cancer, who were treated in our cancer center between 1991-2002, were examined.

The diagnosis of disseminated carcinomatosis of the bone marrow was made in patients with gastric cancer, who also demonstrated: i) an elevation of the serum alkaline phosphatase (ALP) and/or lactate dehydrogenase (LDH) levels, ii) DIC and/or anemia (macro- to normocytic and hyper- to normochromic anemia), and iii) diffuse bone metastases on the bone scintigraphy findings. A bone marrow puncture was performed on 2 patients (cases 5 and 9 in Table I), and metastatic infiltration of atypical epithelial cells (cancer cells) was found in a bone marrow aspiration smear (Fig. 1). Eight 
Table I. Clinicopathological features of nine patients with disseminated carcinomatosis of the bone marrow associated with gastric cancer.

\begin{tabular}{|c|c|c|c|c|c|c|c|c|c|c|c|c|c|}
\hline \multirow[b]{2}{*}{ Case } & \multirow[b]{2}{*}{$\begin{array}{l}\text { Age } \\
\text { (years) }\end{array}$} & \multirow[b]{2}{*}{ Sex } & \multirow[b]{2}{*}{$\begin{array}{l}\mathrm{ALP}^{\mathrm{a}} \\
(\mathrm{IU} / \mathrm{l})\end{array}$} & \multirow[b]{2}{*}{$\begin{array}{l}\mathrm{LDH}^{\mathrm{a}} \\
(\mathrm{IU} / \mathrm{l})\end{array}$} & \multicolumn{2}{|c|}{$\begin{array}{c}\text { Hematological } \\
\text { disorders }^{\mathrm{b}}\end{array}$} & \multirow[b]{2}{*}{$\begin{array}{l}\text { Hyper- } \\
\text { calcemia }^{\mathrm{c}}\end{array}$} & \multirow[b]{2}{*}{$\begin{array}{l}\text { Histological } \\
\text { type }^{\mathrm{d}}\end{array}$} & \multirow[b]{2}{*}{ Therapy $^{\mathrm{e}}$} & \multirow[b]{2}{*}{$\begin{array}{l}\text { Survival } \\
\text { period } \\
(\text { days })^{f}\end{array}$} & \multicolumn{2}{|c|}{$\begin{array}{l}\text { Immunohisto- } \\
\text { chemistry }\end{array}$} & \multirow[b]{2}{*}{$\begin{array}{c}\text { Type } \\
\text { of bone } \\
\text { metastasis }\end{array}$} \\
\hline & & & & & DIC & Anemia & & & & & RANKL & PTHrP & \\
\hline 1 & 52 & F & 475 & 584 & + & + & - & Sig & $\mathrm{CDDP} / 5-\mathrm{FU}$ & 100 & - & - & Osteolytic \\
\hline 2 & 40 & $\mathrm{~F}$ & 395 & 1179 & + & + & - & Mo & BSC & 58 & + & - & Osteolytic \\
\hline 3 & 49 & $\mathrm{~F}$ & 1188 & 310 & + & + & - & Sig & $\mathrm{CDDP} / 5-\mathrm{FU}$ & 240 & + & + & Osteolytic \\
\hline 4 & 45 & $\mathrm{M}$ & 892 & 4820 & + & + & + & Po & $\mathrm{BSC}$ & 43 & + & - & Osteolytic \\
\hline 5 & 65 & $\mathrm{M}$ & 234 & 1915 & - & + & - & Sig & BSC & 10 & + & - & $\mathrm{NE}$ \\
\hline 6 & 63 & $\mathrm{M}$ & 1336 & 1380 & + & + & - & Sig & MTX/5-FU & 190 & - & + & $\mathrm{NE}$ \\
\hline 7 & 50 & M & 168 & 432 & - & + & - & Mo & BSC & 110 & + & - & $\mathrm{NE}$ \\
\hline 8 & 63 & M & 668 & 465 & - & + & + & Po & MTX/5-FU & 220 & - & + & Osteolytic \\
\hline 9 & 40 & $\mathrm{~F}$ & 2404 & 1720 & + & - & - & Mo & $\begin{array}{c}\mathrm{MTX} / 5-\mathrm{FU} \\
\text { BP }\end{array}$ & 330 & + & - & Osteolytic \\
\hline
\end{tabular}

aSerum levels of alkaline phosphatase (ALP) (normal 45-130 IU/l) and lactate dehydrogenase (LDH) (normal 200-370 IU/l) at diagnosis. ${ }^{\mathrm{b} A s s o c i a t e d}$

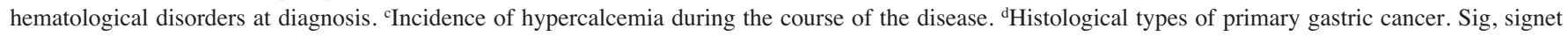
ring cell carcinoma; Po, poorly differentiated adenocarcinoma; Mo, moderately differentiated adenocarcinoma. ${ }^{\circ} \mathrm{CDDP}$, cisplatinum; 5-FU, 5-fluorouracil; MTX, methotrexate; BP, bisphosphonates; BSC, best supportive care. Survival period since the diagnosis of disseminated carcinomatosis of the bone marrow. NE, not examined.

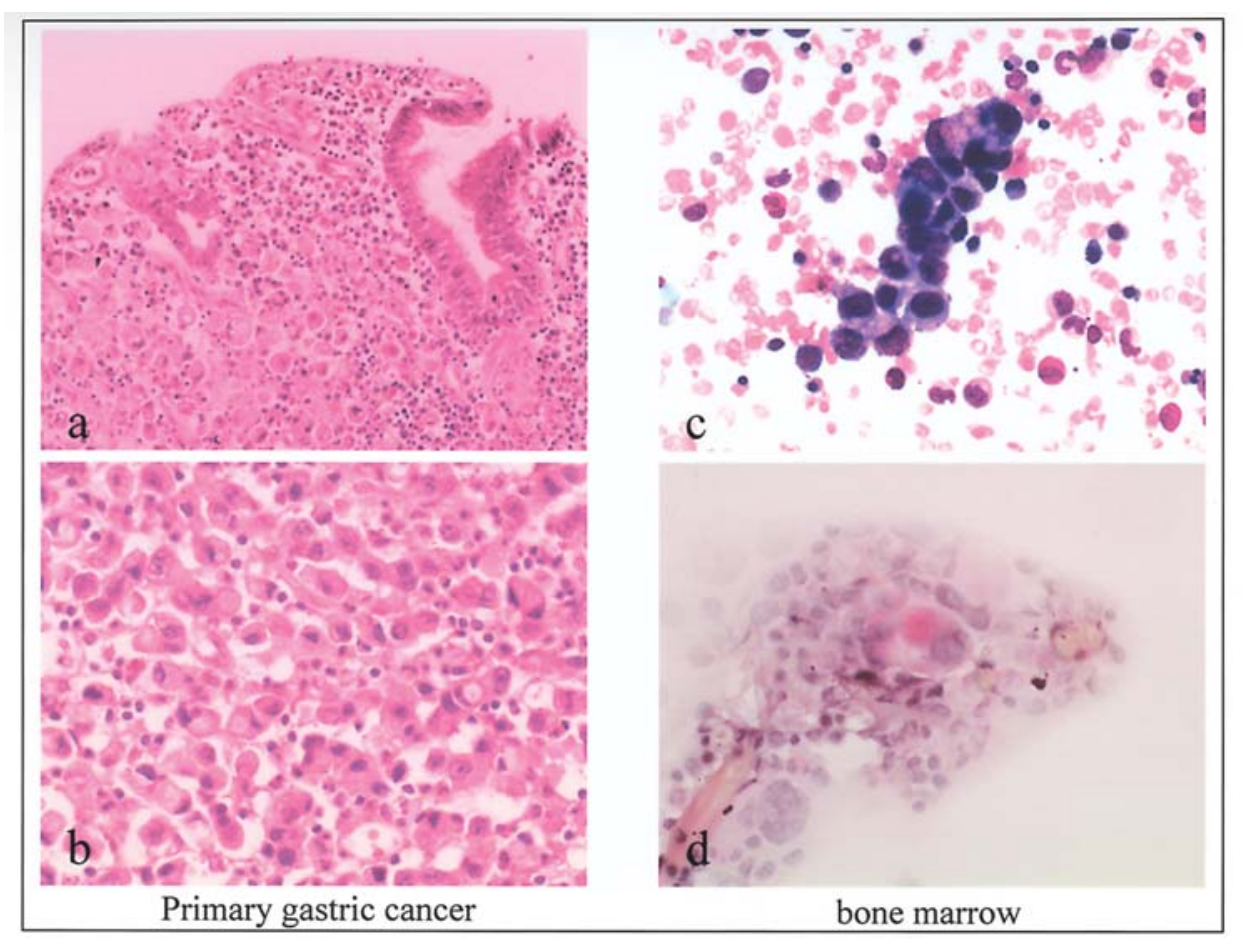

Figure 1. Photomicrographs of a primary gastric cancer specimen (a and b) and bone marrow aspiration smear (c and d) in disseminated carcinomatosis of the bone marrow (case 5). A gastric cancer specimen showed mucosal involvement by signet ring cell carcinoma cells (a, x20; b, x40; hematoxylin and eosin). A bone marrow aspiration smear showed metastatic infiltration by signet ring carcinoma cells (c, x40, Giemsa; d, x40, PAS).

patients had undergone prior surgery for gastric cancer before this condition was identified (cases 1-8 in Table I), and as a result, the diagnosis of gastric cancer was histologically proven in all 8 cases. In one patient (case 9 in Table I), on the other hand, the discovery of this condition preceded the diagnosis of gastric cancer, and the diagnosis was thus made based on the histological findings of the specimens obtained during a gastroendoscopic biopsy. 


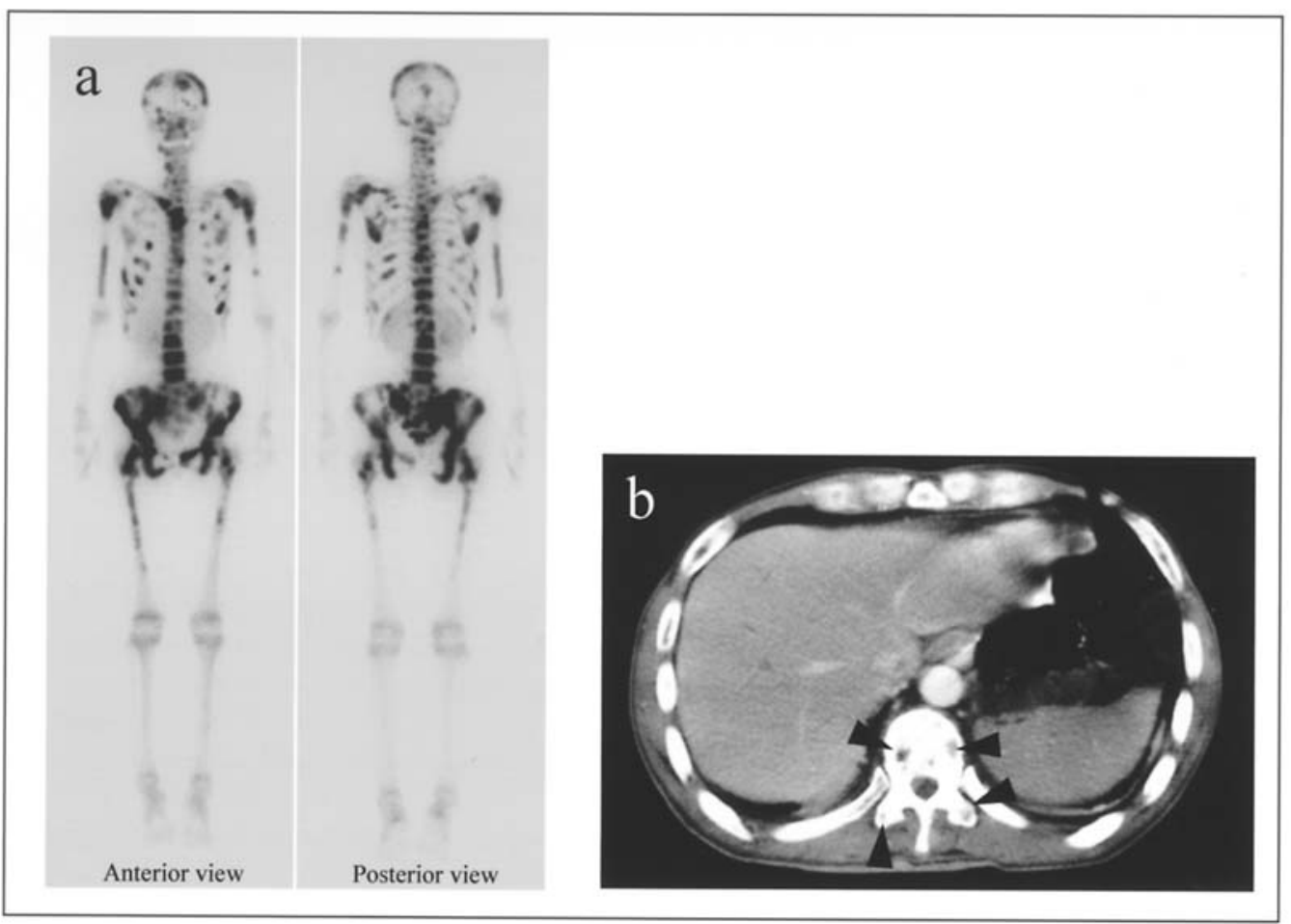

Figure 2. Typical radiological findings of bone lesions in disseminated carcinomatosis of the bone marrow associated with gastric cancer (case 9). The bone scintigraphy showed multiple hot spots throughout the entire skeleton (a). These bone lesions were composed predominantly of osteolytic bone destruction, which was disclosed by CT (b, arrow head).

We determined the clinicopathological features of the 9 patients as follows; age, sex, serum levels of ALP and LDH, presence of hematological disorders (DIC, anemia) at the time this condition was diagnosed, type of bone metastasis, incidence of hypercalcemia during the course of the disease, histological type of gastric cancer, therapy for this condition, and survival period since this condition was diagnosed. These factors are all listed in Table I.

Immunohistochemistry. Immunohistochemical staining for RANKL and PTHrP was performed on gastric cancer specimens obtained at surgery (cases 1-8, Table I) and/or biopsy under gastroendoscopy (case 9, Table I) and bone marrow specimens obtained by bone marrow puncture (cases 5 and 9 , Table I) according to the previously described method (6). We also examined the expression of RANKL and PTHrP immunohistochemically in gastric cancer specimens obtained from 11 patients, in whom no metastasis was found 5 years after operation as a control. The histological types of these patients included 1 signet ring cell carcinoma, 7 poorly differentiated adenocarcinoma and 3 moderately differentiated adenocarcinoma. This composition of the histological types of the patients were similar to those of the gastric cancer patients who presented disseminated carcinomatosis of the bone marrow.

Briefly, the sections $(4 \mu \mathrm{m})$ were de-waxed with xylene and rehydrated through a graded series of ethanol. After inhibition of endogenous peroxidase, the sections were incubated with primary antibodies at $4^{\circ} \mathrm{C}$ overnight, followed by incubation with biotinylated link antibody and peroxidaselabeled streptavidin (Nichirei, Tokyo, Japan) at room temperature for $20 \mathrm{~min}$. The sections were then reacted in 3,3'- diaminobenzidine tetrahydrochloride (DAB), and then were counterstained with hematoxylin. We used goat polyclonal antibodies against RANKL (sc-7627, 1:50, Santa Cruz Biotechnology, Santa Cruz, CA) and PTHrP (sc-9680, 1:50, Santa Cruz Biotechnology) as a primary antibody. Tissue samples from giant cell tumors of the bone and PTHrP-producing lung cancer were used as positive controls for RANKL and PTHrP, respectively, while sections incubated with PBS instead of the primary antibodies were used as negative controls for both.

Statistics. The statistical analysis was performed using Welch's unpaired t-test, where a value of $\mathrm{p}<0.05$ was considered to be statistically significant.

\section{Results}

The characteristic clinicopathological features of disseminated carcinomatosis of the bone marrow associated with gastric cancer. The clinicopathological features of the 9 patients with gastric cancer demonstrating disseminated carcinomatosis of the bone marrow are listed in Table I.

The nine patients with this condition included 5 males and 4 females with a median age of 52 years, which was younger than that for the majority of gastric cancer patients. An elevation in the serum ALP and LDH levels was a characteristic clinical finding, which is important for making the diagnosis, and the elevated ALP level was mainly related to bone origin. Wide-spread bone metastases to almost the entire skeleton were detected by bone scintigraphy (Fig. 2a), and the types of bone metastasis included osteolytic bone destruction in all 6 patients whose bone lesions were evaluated 


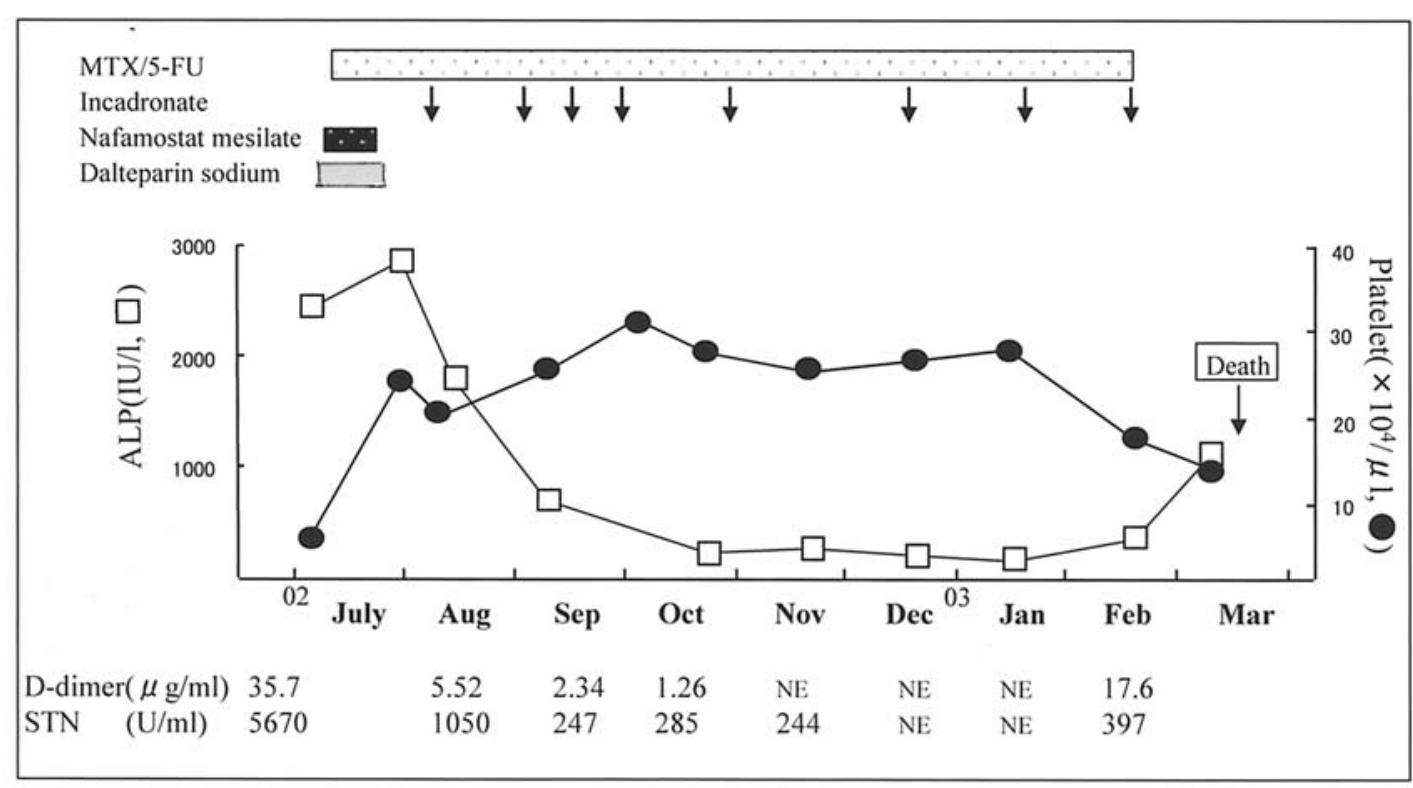

(NE, not examined)

Figure 3. The treatment for disseminated carcinomatosis of the bone marrow associated with gastric cancer in case 9 . The platelet count increased to the

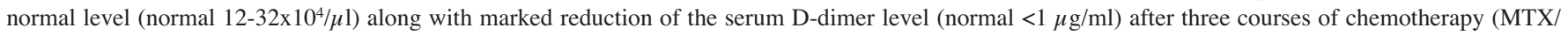
5-FU) and the treatment for DIC (Nafamostat mesilate, Dalteparin sodium). However, the serum levels of ALP, which represented the extent of bone lesions, and a tumor marker, STN, still remained extremely high. Then, a bone-targeted therapy using bisphosphonates (incadronate) was started in addition to chemotherapy, which resulted in a rapid reduction of serum ALP (normal 45-130 IU/l) and STN (normal <45 U/ml) levels.

by plain radiography, $\mathrm{CT}$ and/or magnetic resonance imaging (MRI) (Fig. 2b). DIC, the most common hematological complication associated with disseminated carcinomatosis of the bone marrow, was found in 6 of 9 patients. In the remaining 3 patients without DIC, macro- to normo-cytic and hyperto normo-chromic anemia was noted as a hematological complication. This type of anemia together with an elevation of the serum LDH level suggest the possibility that the anemia had been caused by a diffuse infiltration of cancer cells into the bone marrow. Hypercalcemia is sometimes associated with multiple bone metastases in cancer patients. In the present study, however, only two of the 9 patients developed hypercalcemia during the course of the disease in spite of a widespread expansion of bone metastases.

The histological types of gastric cancer included 4 signet ring cell carcinomas, 2 poorly differentiated adenocarcinomas and 3 moderately differentiated adenocarcinomas. In the 3 gastric cancers with moderately differentiated adenocarcinoma, however, a poorly differentiated component was found in some parts of the gastric cancer in one patient. Chemotherapy [cisplatinum (CDDP)/5-fluorouracil (5-FU) or methotrexate (MTX)/5-FU] was performed in 4 patients (cases 1, 3, 6, 8; Table I), and the mean $( \pm \mathrm{SD})$ survival period of these patients was $187 \pm 62$ days. In the 4 patients without chemotherapy (cases 2, 4, 5, 7; Table I), on the other hand, the mean $( \pm \mathrm{SD})$ survival period was $55 \pm 42$ days, which was significantly shorter than that of the patients with chemotherapy $(\mathrm{p}<0.02)$. In the remaining one patient (case 9, Table I) who was treated most recently in our cancer center, a bone-targeted therapy using bisphosphonates (incadronate) was performed in addition to chemotherapy (MTX/5-FU). The clinical course of this patient is shown in Fig. 3. Chemotherapy for gastric cancer and treatment for DIC were started first, and DIC was no longer seen when the three courses of these treatments had finished. However, serum ALP levels, which represented the extent of bone metastases, still remained at a high level, thus, a bone-targeted therapy using bisphosphonates (incadronate) was started in addition to chemotherapy. As a result, serum ALP levels began to reduce along with the serum levels of a tumor marker, sialyl Tn-antigen (STN), and the reduced levels of serum ALP and STN were maintained for 6 months. However, the patient then died because of progression of DIC.

Expression of RANKL and PTHrP in gastric cancer. We performed immunohistochemistry for RANKL and PTHrP to identify any factors related to pathogenesis regarding the development of bone lesions in disseminated carcinomatosis of the bone marrow. RANKL showed positive staining in the gastric cancer tissue specimens from 6 of 9 patients (Fig. 4a and b), while PTHrP was positively stained in the same specimens in 3 of 9 patients (data not shown). In the bone marrow specimens, on the other hand, positive staining for RANKL was found in the gastric cancer cells invading the bone marrow in 2 patients examined (Fig. 4c and d), whereas PTHrP was not positively stained in these cells (data not shown).

We also examined the expression of these proteins immunohistochemically in the gastric cancer specimens without any metastasis as a control. RANKL was positively stained in only one of 11 patients, while PTHrP was positively stained in 3 of 11 patients (data not shown).

\section{Discussion}

Disseminated carcinomatosis of the bone marrow is accompanied by solid tumors and gastric cancer accounts for the majority of such cases (1-3), although the incidence of this 


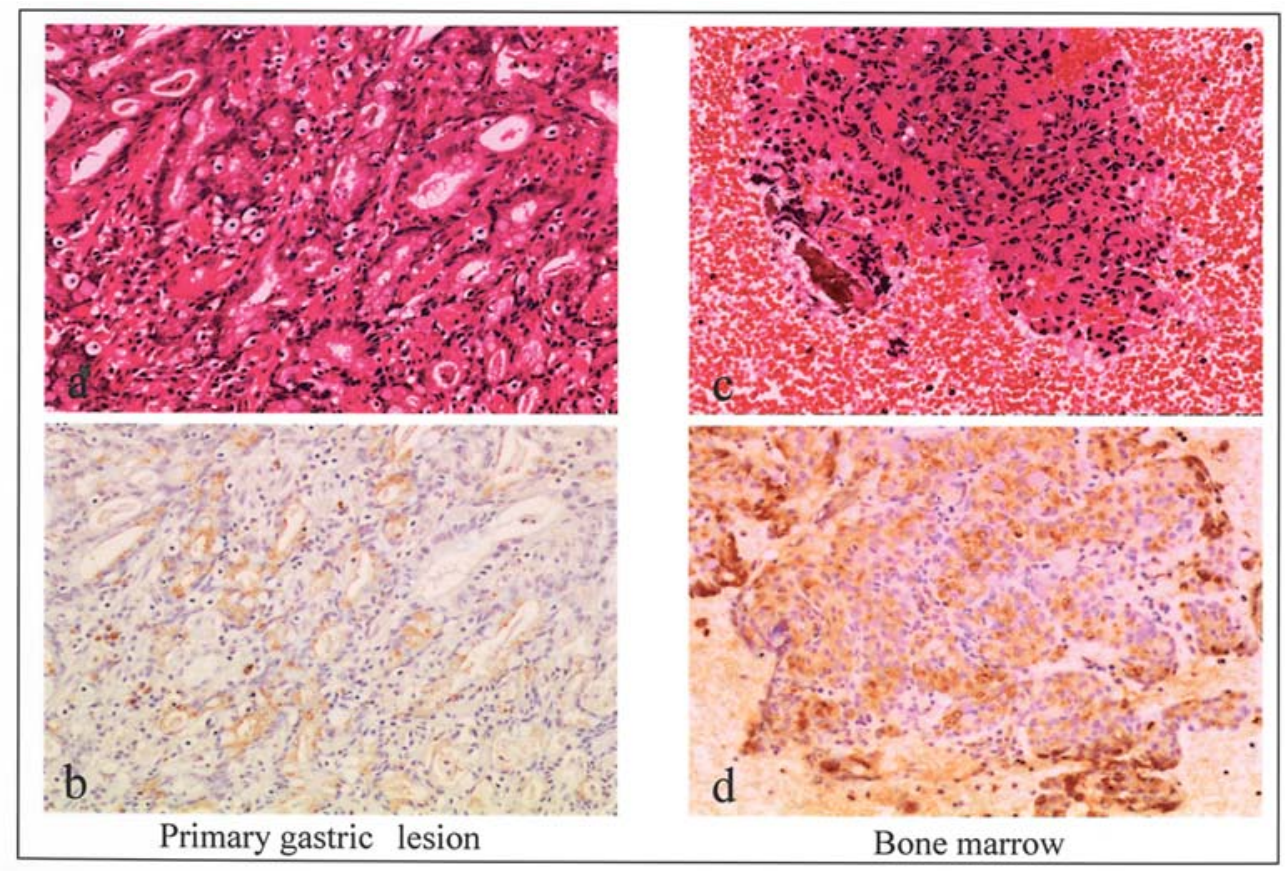

Figure 4. Representatives of immunohistochemistry for RANKL in gastric cancer demonstrating disseminated carcinomatosis of the bone marrow (case 9). (a) The findings for hematoxylin and eosin staining of gastric cancer show moderately differentiated adenocarcinoma (x20). (b) Immunohistochemistry for RANKL in a serial section of the same specimen in (a). RANKL shows positive staining predominantly in the cytoplasm and plasma membrane of moderately differentiated adenocarcinoma cells (x20). (c) The findings for hematoxylin and eosin staining of a bone marrow aspiration smear show infiltration of atypical epithelial cells indicating metastasis from known gastric cancer (x20). (d) Immunohistochemistry for RANKL in a serial section of the same specimen in (c). RANKL shows positive staining predominantly in the cytoplasm and plasma membrane of metastatic gastric cancer cells as is seen in the primary lesion (b) (x20).

condition is rare. In this condition, cancer cells diffusely infiltrate the bone marrow followed by explosive proliferation, which causes bone destruction and hematological complications. However, the pathogenesis for the development of bone lesions is still poorly understood. In the present study, we examined the characteristic clinicopathological features of disseminated carcinomatosis of the bone marrow from gastric cancer based on the clinical findings of our patients. The typical features included a younger patient age, an elevation in the serum ALP and/or LDH, extensive bone metastases with osteolytic bone destruction, a low incidence of hypercalcemia and histological types of gastric cancer with signet ring cell carcinoma or poorly differentiated adenocarcinoma. Only a few cases in a case report are available regarding disseminated carcinomatosis of the bone marrow. Morimatsu et al (7) examined 11 autopsy cases of gastric cancer patients with diffuse bone marrow metastasis, and found the patients to be relatively young (mean 47 years) while 9 of 11 cases had a signet ring cell component. These results are consistent with the findings in the present study.

The prognosis of these patients was poor regardless of the treatment. However, the survival period of the patients treated with chemotherapy was significantly longer than that for those without chemotherapy. Furthermore, the survival period of the patient treated with bisphosphonates in addition to chemotherapy was longer than that of the patients with chemotherapy alone although only one case was treated with bisphosphonates. Chemotherapy has been reported to improve the prognosis of metastatic gastric cancer (8). In addition, a new approach for the treatment for bone metastasis using bisphosphonates has been established (9). Bisphosphonates have an anti-proliferative effect on cancer cells by decreasing the supply of growth factors from the bone through the inhibition of bone resorption $(10,11)$. Currently, nitrogencontaining bisphosphonates have been shown to inhibit cancer cell proliferation directly through the inhibition of the mevalonate signaling pathway (12-14). Bisphosphonates seem to be included in the treatment arm in combination with chemotherapy against disseminated carcinomatosis of the bone marrow.

To elucidate the pathogenesis of the development of bone lesions in disseminated carcinomatosis of the bone marrow, we immunohistochemically examined the expression of RANKL and PTHrP, which are master regulators of osteoclast differentiation and activation, in gastric cancer tissue and bone marrow specimens of these patients. Among various cancers, the expression of RANKL was found in myeloma $(15,16)$ and/or ATL cells (17), whereas such expression was rare in solid tumors (18). In the present study, we demonstrated the high incicence of the expression of RANKL in gastric cancer cells, which extensively invaded the bone compared to that in gastric cancer without any metastasis. To our knowledge, this is the first study demonstrating the expression of RANKL in gastric cancer cells. This finding raises the possibility that gastric cancer cells act directly on osteoclast precursor cells via RANKL to promote osteoclast formation. This finding seems to imply the mechanism responsible for developing bone lesions in disseminated carcinomatosis of the bone marrow. We also found the expression of PTHrP in the gastric cancer tissue specimens obtained from patients with disseminated carcinomatosis of the bone marrow. However, its incidence was lower than that of RANKL, and similar to that of PTHrP in the control patients without any metastasis. PTHrP expression has been identified within tumor cells in 71 of 92 
patients with gastric cancer (19). PTHrP expression might not be a specific event for the gastric cancer associated with disseminated carcinomatosis of the bone marrow.

In summary, RANKL, which is expressed in gastric cancer cells, is considered to play an important role in the development of bone lesions in disseminated carcinomatosis of the bone marrow by gastric cancer. Bone-targeted therapy using bisphosphonates, in addition to chemotherapy, is recommended as the treatment of choice for patients with this condition.

\section{Acknowledgements}

This study was supported in part by Health and Labor Science Research Grants [Research On Human Genome, Tissue Engineering Food Biotechnology (H15-Genome-002)] from the Ministry of Health, Labor and Welfare, Japan.

\section{References}

1. Pasquini E, Gianni L, Aitini E, et al: Acute disseminated intravascular coagulation syndrome in cancer patients. Oncology 52: 505-508, 1995.

2. Crivellari D, Carbone A, Sigon R, et al: Gastric cancer with bone marrow invasion at presentation: case-report and review of the literature. Tumori 81: 74-76, 1995.

3. Noda N, Sano T, Shirao K, et al: A case of bone marrow recurrence from gastric carcinoma after a nine-year disease-free interval. Jpn J Clin Oncol 26: 472-475, 1996.

4. Hofbauer LC, Neubauer A and Heufelder AE: Receptor activator of nuclear factor-kappaB ligand and osteoprotegerin: potential implications for the pathogenesis and treatment of malignant bone diseases. Cancer 92: 460-470, 2001.

5. Kitazawa $\mathrm{S}$ and Kitazawa R: RANK ligand is a prerequisite for cancer-associated osteolytic lesions. J Pathol 198: 228-236, 2002.

6. Oda Y, Takahira T, Kawaguchi K, et al: Alterations of cell cycle regulators in myxofibrosarcoma with special emphasis on their prognostic implications. Human Pathol 34: 1035-1042, 2003.
7. Morimatsu M, Shirouzu K, Irie K, et al: Gross and microscopic characteristics of stomach cancer with microangiopathic hemolytic anemia and/or disseminated intravascular coagulopathy. Acta Pathol Jpn 35: 809-822, 1985.

8. Yoshida M, Ohtsu A, Boku N, et al: Long-term survival and prognostic factors in patients with metastatic gastric cancers treated with chemotherapy in the Japan Clinical Oncology Group (JCOG) study. Jpn J Clin Oncol 34: 654-659, 2004.

9. Berenson JR, Rosen LS, Howell A, et al: Zoledronic acid reduces skeletal-related events in patients with osteolytic metastases. Cancer 91: 1191-1200, 2001.

10. Carano A, Teitelbaum SL, Konsek JD, et al: Bisphosphonates directly inhibit the bone resorption activity of isolated avian osteoclasts in vitro. J Clin Invest 85: 456-461, 1990.

11. Hughes DE, Wright KR, Uy HL, et al: Bisphosphonates promote apoptosis in murine osteoclasts in vitro and in vivo. $\mathrm{J}$ Bone Miner Res 10: 1478-1487, 1995.

12. Shipman CM, Croucher PI, Russell RG, et al: The bisphosphonate incadronate (YM175) causes apoptosis of human myeloma cells in vitro by inhibiting the mevalonate pathway. Cancer Res 58: 5294-5297, 1998.

13. Senaratne SG, Pirianov G, Mansi JL, et al: Bisphosphonates induce apoptosis in human breast cancer cell lines. Br J Cancer 82: 1459-1468, 2000.

14. Lee MV, Fong EM, Singer FR and Guenette RS: Bisphosphonate treatment inhibits the growth of prostate cancer cells. Cancer Res 61: 2602-2608, 2001

15. Sezer O, Heider U, Jakob C, et al: Human bone marrow myeloma cells express RANKL. J Clin Oncol 20: 353-354, 2002.

16. Farrugia AN, Atkins GJ, To LB, et al: Receptor activator of nuclear factor-kappaB ligand expression by human myeloma cells mediates osteoclast formation in vitro and correlates with bone destruction in vivo. Cancer Res 63: 5438-5445, 2003.

17. Nosaka K, Miyamoto T, Sakai T, et al: Mechanism of hypercalcemia in adult T-cell leukemia: overexpression of receptor activator of nuclear factor kappaB ligand on adult T-cell leukemia cells. Blood 99: 634-640, 2002.

18. Chikatsu N, Takeuchi Y, Tamura Y, et al: Interactions between cancer and bone marrow cells induce osteoclast differentiation factor expression and osteoclast-like cell formation in vitro. Biochem Biophys Res Commun 267: 632-637, 2000.

19. Alipov GK, Ito M, Nakashima M, et al: Expression of parathyroid hormone related peptide (PTHrP) in gastric tumours. J Pathol 182: 174-179, 1997. 